\title{
The second millisecond pulsar
}

\section{On the principle that no celestial object is unique, the discovery of another millisecond pulsar is overdue. That reported on page 417 has an unexpected interest.}

WHEN the first millisecond pulsating star (PSR1937 + 214) was discovered more than a year ago, there were two contrasting views of what might happen next: either the $1.5-\mathrm{ms}$ rotating neutron star was such an exceptional object, so near the edge of instability perhaps, that it would prove to be an isolated phenomenon, or the late discovery of this quickly spinning object was a sign that radioastronomers had previously neglected objects in this range, so that the first discovery would quickly be followed by several others. The truth is somewhere in between. Far from a flood of new discoveries of pulsars with periods in the millisecond range, there has been just one - that reported by Boriakoff et al. on page 417 . Millisecond pulsars are plainly uncommon though not exceptional objects. The new pulsar has the convenience of being the principal member of a binary system, which explains why the report of its discovery comes complete with no fewer than half a dozen similar explanations of how it may have been formed.

Even so, it is worth recalling the enormity of what needs to be explained. All pulsars are neutron stars, and nobody has seriously quarrelled with Chandrasekhar's estimate that it needs a mass 1.3 times that of the Sun to make a neutron star rather than a degenerate dwarf in which atomic nuclei retain their identity. Most pulsars have periods measured in seconds, which is explicable only because of the compactness and tensile strength of neutron matter (derived from the strong nuclear forces holding them together), which enable these stars to survive the stresses of rapid rotation. As it happens, the rotational speed of a neutron star is limited not so much by its tensile strength as by the rate at which a rapidly spinning object would lose energy by means of gravitational waves caused by readjustments of its shape.

Speed apart, the first millisecond pulsar had two other exceptional properties. The rate of deceleration of the spin, measurable enough, is small compared with the decelerations of more conventional pulsars. But since the deceleration of the rotation of a pulsar is a consequence of the interaction of matter with its magnetic field, the strength of the magnetic field of this quickly spinning pulsar must also be small compared with that of more conventional pulsating stars. So far as can be told from the data now published, the new pulsar PSR $1953+29$, with a rotational period of $6.1 \mathrm{~ms}$, shares the same unex- pected properties - small deceleration and a relatively small magnetic field (estimated at about $10^{10}$ gauss).

The fact that the new object is a partner in a binary system is the starting point for the accompanying accounts of how it may have arisen. Indeed, the new pulsar is only the fourth to have been found within a binary system, much less than the proportion (more than a half) of ordinary stars included within binary systems. No doubt the explanation is that the occurrence of a supernova explosion within such a system will often send the residual neutron star scurrying away. The consequence, however, is that it has been possible to glean something of the mass of the system from half a period's observation of the Doppler shift of the radio pulsation. The conclusion that the orbit is virtually circular is only tentative. The conclusion that the pulsar is more massive than its companion is not compelled by the data but is likely to be true. The accompanying explanations agree that the companion is now a white dwarf with a mass about 0.3 that of the Sun.

The explanations of the origin of the 6.1ms pulsar follow similar lines. The common notion is that the now unseen component of the binary system has within the comparatively recent past (ten million years?) gone through the giant phase in the normal evolution of a star. To begin with, the orbit was much smaller than at present. When most of the internal hydrogen had been converted into helium, the star became a giant, burning hydrogen only in its envelope which by then, in any case, would have reached beyond the gravitational equipotential point between the two stars. Material would then have been transferred from the companion star to the primary together with angular momentum. So, the argument goes, the end result would be that now observed - a rapidly pulsating neutron star whose mass is comparable with that of the Sun and a companion in a nearly circular orbit that has lost all but the helium core with which it embarked on its giant phase. It seems to be agreed that if the dwarf continues to burn hydrogen in its envelope, it should be two orders of magnitude more luminous than the Sun and thus just on the limit of detectability even at a distance of 3,500 parsecs.

By sheer good luck, Helfand et al. (see page 423 ) have important information on this point. The new pulsar happens to be within one minute of arc from another from which X-ray emission was sought during the lifespan of the Einstein X-ray satellite, with the result that it has been possible to use data to set an upper limit of the X-ray emission from the new system. The upshot of the argument is that the most likely progenitor of the pulsar was not a neutron star as such but a white dwarf which, by accreting matter from its oncegiant companion, exceeded the critical mass of 1.3 times the solar mass, pushing it over the threshold separating dwarfs from neutron stars.

Everybody seems to agree that whatever the original condition of the system, it must recently have gone through a phase in which it would have been recognizable as a low-mass X-ray binary of the kind described by Virginia Trimble in her recent fieldguide to binary stars (Nature 303, 137; 1983). Ruderman and Shaham carry the argument a step further in their account of the origin of the apparently isolated $1.5-\mathrm{ms}$ pulsar. They conclude that there is a high chance (determined by the precise details of the orbit and the relative masses of the two stars) that in a binary system consisting of a white dwarf primary and a secondary that has become a giant, tidal forces may so disrupt the secondary star when the mass transfer has gone some way that it will either be totally fragmented or reduced in size to an undetectable planet the size of, say, Saturn.

All this is good clean fun of the kind that keeps astrophysics entertained - and entertaining. The discovery of the $6.1-\mathrm{ms}$ pulsar does, however, reinforce the opinion that has been growing for some time that there are two kinds of pulsars - those formed in supernova explosions (usually with modest rotational speeds) and those formed by the accretion of matter onto white dwarfs or other degenerate objects. Pulsars of the former class have relatively small rotational speeds (with periods measured in seconds), large velocities perpendicular to the galactic disk and strong magnetic fields. The second class, consisting of pulsars formed by accretion onto a degenerate star, are represented by the two millisecond pulsars so far found and possibly by some of those with smaller rotational speeds. Telling which are which will always be difficult at the borderline. Looking for X-ray binaries that may be nascent systems like PSR $1953+29$ should, however, be rewarding - Trimble explains that there is a host of candidates.

John Maddox 